\title{
Effect of different exercise rehabilitation programs with body weight reduction on reducing the time of injured limb efficient restoration before and after low knee osteotomy surgery
}

\author{
Waleed M. HEDYA \\ Assistant Professor of Biological Sciences and Sports Health, \\ Faculty of Physical Education for Men, \\ Matrouh University, Egypt.
}

\section{Introduction}

Lower limb osteotomies are a significant area in orthopaedic surgery and research, interested largely by a cumulative occurrence and distribution of osteoarthritis and other medical problems (Gao et al., 2019).

In a normally aligned knee, the center of pressure passes somewhat to the medial side during extension. During flexion, the center of pressure is more medial. Axial varus malalignment leads to an abnormal load distribution across the medial compartment, pathologically disturbing both tibiofemoral osteochondral units and the medial meniscus. A $4 \%$ to $6 \%$ rise in varus malalignment significantly raises loading (up to $20 \%$ ) in the medial compartment, disturbing the articular cartilage, the subchondral bone and the medial meniscus (Ziegler et al. 2015).

A knee osteotomy is a surgical operation whereby a bone is cut to shorten, lengthen or change its alignment to increase the stability of the knee and reduce the effects of arthritis (Brouwer et al., 2014).

Knee surgery gives acceptable results in patients with advanced degenerative variations in the knee. The most public indications for this surgical process are osteoarthritis, rheumatoid arthritis and knee stiffness (Nawghare and Brooks 2013).

Satisfaction after knee surgery ranges between 75 and $90 \%$. From a surgical point of view, joint line elevation is had a negative effect on surgical outcome, since it changes the biomechanics of the knee. By altering the center of rotation of the knee, the isometric of the medial collateral ligament is altered, with mid-flexion instability as a result. Moreover, as a result of the elevated joint line, the posterior condylar offset is likely to be condensed, which negatively influences flexion angle and the extensor mechanism strength, and resolves in mid-flexion instability (Lieshout et al., 2018).

The pain that associated with knee surgery is often activity related (Syed and Wani 2014; Eitner et al., 2017). However, pain is not the only consequence of knee surgery experienced by patients. Pain is related to 
function, with physical movements triggering pain, while pain, in turn, causes restrictions in physical function (Castrogiovanni and Musumeci 2017).

The haematoma in the gap resulted from surgery is replaced by connective tissue in period ranges from four to six weeks (Fernandes et al., 2011), which provides further callus formation and ossification, visible by three months after surgery (Brinkman et al., 2008).

Postoperative therapy is done to reduce swelling. Starting on the first postoperative day, partial weight-bearing from $15 \mathrm{~kg}$ to $20 \mathrm{~kg}$ of the body weight is permitted. From four to six weeks the amount of weight-bearing allowed is based on the amount of pain, although after six weeks full weightbearing is permitted. A full range of active and passive movement is encouraged, and started with the help of a physiotherapist (Brinkman et al., 2008).

Many people with knee surgery are capable of performing independent land based exercise programs with an objective of maintain the health, improving knee function, and decreasing the risk of inactivity that associated with muscle disorder (Sisto and Malanga 2006). There is some evidence signifying that mechanical unweighting exercise may be more effective than weight bearing exercise in patients with medial knee problems (Bennell et al., 2019).

Water based exercise may be an excellent alternative to land based exercise for individuals who have joint pain. It reduces the weight that joints, bones and muscles have to bear. Pressure of water consider as good reducing factor for swelling and load on painful joints while it is enhancing muscle relaxation. Aquatherapy causes many effects on the musculoskeletal system. The effects are caused by the compressive effects of immersion as well as reflex regulation of blood vessel tone. During immersion, it is expected that most of the improved cardiac output is redistributed to skin and muscle (Mooventhan and Nivethitha 2014). Some studies documented that patients must wait until two weeks after surgery to allow for wounds to heal and this may be slightly varied according to quality of individuals healing (Villalta and Peiris 2013). Depending on this rationale, the knee rehabilitation time may be delaying.

The rehabilitation with water based and land base exercises decreased level of pain, improved leg strength, flexibility and balance of the affected knee (Adnan et al., 2014).

The present study aimed at assessing the effect of different exercise rehabilitation programs with body reducing weight on reducing the time of injured limb efficient restoration before and after low knee osteotomy surgery. 


\section{Materials and methods}

\subsection{Study sample}

The study sample comprised twenty four patients (46-62 years-old) diagnosed with need for injured limb efficient restoration after low knee osteotomy surgery, had no issues of blood pressure, pulse, and breathing, they also had no consciousness or sensory disorders.

\subsection{Study domains}

\subsubsection{Study time}

Period: From February 2017 to May 2019.

\subsubsection{Study place}

Study was performed at health and sport scientific center Port Said.

\subsubsection{Study approach}

Study was performed using the experimental approach.

\subsection{Experimental setup and working}

The study sample was divided randomly to three experimental groups (each group composed of eight individuals), skew test was performed between different experimental groups before the knee surgery and treating programs for symmetry, it was between \pm 3 .

The performed therapeutic exercise was based on recognized of impairments related to the knee osteoarthritis before and after the surgery, intensive mainly on strengthening, functional balance and muscle control.

Preoperative exercise was divided to two types: The first type was performed generally with all study groups focused on lower limb strengthening, with attention for strengthening of the quadriceps muscles. Before performing the main strength training program, subjects performed 20 minutes of ergometer bike. After the warm-up, patient applies a series of lower-body strengthening exercises, short arc extension, wall squat, leg press, isometric leg extension, and leg curls as well as other exercises according to the patient tolerance as showed in (Table 2.1), all study participates were received 4-week preoperative program (Kim et al., 2015). The patient received 6 sessions /week.

While the second type was designed specifically for each experimental group as following: The first experimental group specific treating program was done using the mechanical unweighting, while the second experimental group treating program was done using the aquatherapy, and the last experimental group was applied the two previous treating program starting with mechanical unweighting program followed using the aquatherapy program. 
Postoperative exercises use the traditional program for all groups were done immediately after surgery plus the suggested programs for each experimental group separately with different starting time.

Table 2.1: Preoperative exercise traditional program that all groups were done.

\begin{tabular}{|c|c|c|}
\hline $\begin{array}{c}\text { Exercises } \\
\text { category }\end{array}$ & Exercises & $\begin{array}{c}\text { Repetitions } \\
\text { and set }\end{array}$ \\
\hline Cardio exercise & Ergometer bike & $(15-25) \mathrm{min}$ \\
\hline $\begin{array}{c}\text { Range of motion } \\
\text { exercise }\end{array}$ & $\begin{array}{c}\text { Seat flexion/extension then } \\
\text { wall slides }\end{array}$ & $(8-12) \mathrm{min}$ \\
\hline $\begin{array}{c}\text { Open-chain } \\
\text { strengthening }\end{array}$ & Short arc extension & $10 \times 2-12 \times 3$ \\
\cline { 2 - 3 } & Straight leg raises & $10 \times 2-12 \times 3$ \\
\hline Leg curl (isometric) & $10 \times 2-12 \times 3$ \\
\hline $\begin{array}{c}\text { Close-chain } \\
\text { strengthening }\end{array}$ & Leg extension (ROM $30-80^{\circ}$ ) & $10 \times 2-12 \times 3$ \\
\cline { 2 - 3 } & Leg press & $10 \times 2-15 \times 3$ \\
\hline \multirow{2}{*}{$\begin{array}{c}\text { Balance/Propriocep } \\
\text { tion }\end{array}$} & Single-leg standing & $\begin{array}{c}30 \times 2-15 \times 3 \\
\text { sec } \times 3-30 \\
\sec \times 5\end{array}$ \\
\cline { 2 - 3 } & Balance board & $\begin{array}{c}30 \mathrm{sec} \times 3-30 \\
\sec \times 5\end{array}$ \\
\hline
\end{tabular}

\subsubsection{Mechanical unweighting program}

Done for four weeks before the surgery, then returned from the fourth week and ended at the twenty week after the surgery.

The patient received 5sessions/week, at least 30 minutes of moderate physical activity (Adams et al., 2013).

Mechanical unloading during exercise was made by the use of a Zuni exercise system. This system used a harness suspended from a cable equipped with a tensiometer that permitted a preset weight reduction to be maintained during unweighting exercises and enabled subjects to perform exercises at $0 \%, 20 \%$, and $40 \%$ of body weight support (BWS) (Mangione et al., 1996). The exercises are summarized in Table 2.2.

Table 2.2: Mechanical un-weighting program from standing position that the first experimental group was done.

\begin{tabular}{|c|c|}
\hline Exercises & $\begin{array}{c}\text { Repetitions and } \\
\text { set }\end{array}$ \\
\hline Alternating knee raises & $10 \times 2-3$ \\
\hline Alternating leg raises & $10 \times 2-3$ \\
\hline
\end{tabular}




\begin{tabular}{|c|c|}
\hline $\begin{array}{c}\text { Alternating raised leg } \\
\text { swings }\end{array}$ & $20 \times 2-3$ \\
\hline Side leg raises & $20 \times 2-3$ \\
\hline Rotate leg raises & $10-20 \times 2-3$ \\
\hline Single leg circle & $20 \times 2-3$ \\
\hline Raised knee extension & $2-3 \times 15$ \\
\hline Knee stabilization & $2-3 \times 15$ \\
\hline Heel and toe raises & $2-3 \times 20$ \\
\hline $\begin{array}{c}\text { Standing hamstring } \\
\text { stretch }\end{array}$ & $15-30$ sec $\times 2-3$ \\
\hline Quadriceps stretch & $15-30$ sec $\times 2-3$ \\
\hline Wide single leg squats & $10 \times 2-3$ \\
\hline Cross leg side title & 10 sec $\times 2-3$ \\
\hline Altering split squats & $10 \times 2-3$ \\
\hline $\begin{array}{c}\text { Each leg hamstring } \\
\text { stretch }\end{array}$ & 15 sec $\times 2-3$ \\
\hline Set-up & $10-15 \times 2-3$ \\
\hline Butt kicks & $10-15 \times 2-3$ \\
\hline
\end{tabular}

\subsubsection{The aquatherapy program}

Done for four weeks before the low knee osteotomy surgery, then returned from the sixth week and ended at the twenty two week after surgery.

The program comprised functional weight-bearing and progressive exercises (Table 2.3) provided twice weekly (45-60 minutes each) (Hinman et al., 2006; Sarsak 2018).

\subsubsection{Combined effect of the two previous programs}

Done for four weeks before the low knee osteotomy surgery, then returned from the fourth week and ended at the seventeenth week after surgery.

Table 2.3: Aquatherapy program that the second experimental group was done.

\begin{tabular}{|c|c|c|c|}
\hline Phase & Exercises & $\begin{array}{c}\text { Sets and } \\
\text { repetitions }\end{array}$ & $\begin{array}{c}\text { Walki } \\
\text { ng }\end{array}$ \\
\hline 1 & $\begin{array}{l}\text { 1. Double-leg squats } \\
\text { 2. Double-leg calf raises } \\
\text { 3. Dynamic lunge }\end{array}$ & $\begin{array}{l}2 \times 10 \\
2 \times 10 \\
2 \times 10\end{array}$ & $6 \min$ \\
\hline 2 & As for phase 1 & As for phase & $8 \mathrm{~min}$ \\
\hline 3 & As for phase 1, plus: & $\begin{array}{l}2 \times 10 \\
2 \times 10 \\
\end{array}$ & $\begin{array}{c}10 \\
\min \end{array}$ \\
\hline
\end{tabular}




\begin{tabular}{|c|c|c|c|}
\hline & $\begin{array}{l}\text { 4. Single-leg stance, contralateral } \\
\text { knee flexion/extension } \\
\text { 5. Single-leg stance, contralateral } \\
\text { hip abduction/adduction } \\
\text { 6. Single-leg stance, contralateral } \\
\text { hip hitching }\end{array}$ & $2 \times 10$ & \\
\hline 4 & $\begin{array}{l}\text { 1. Single-leg squats } \\
\text { 2. Sincle-leg calf raises } \\
\text { 3. Dynamic lunge } \\
\text { Plus exercises } \\
4,5 \text {, and } 6 \text { from phase } 3\end{array}$ & $\begin{array}{l}2 \times 10 \\
2 \times 10 \\
2 \times 10\end{array}$ & $\begin{array}{c}10 \\
\min \end{array}$ \\
\hline 5 & $\begin{array}{l}\text { As for phase 4, plus: } \\
\text { 7. Step-ups }\end{array}$ & $2 \times 10$ & $\begin{array}{c}10 \\
\min \end{array}$ \\
\hline 6 & $\begin{array}{l}\text { As for phase } 5 \text {, but modify: } \\
\text { 7. Step-downs }\end{array}$ & $2 \times 10$ & $\begin{array}{c}10 \\
\min \end{array}$ \\
\hline 7 & $\begin{array}{l}\text { As for phase } 6 \text {, but for exercises } \\
4 \text { and } 5 \text {, increase speed } \\
\text { (resistance) of moving leg as } \\
\text { able }\end{array}$ & $\begin{array}{l}2 \times 10 \text { followed } \\
\text { by } 1 \times 15\end{array}$ & $\begin{array}{c}10 \\
\min \end{array}$ \\
\hline 8 & As for phase 7 & $3 \times 10$ & $\begin{array}{c}10 \\
\min \end{array}$ \\
\hline 9 & As for phase 7 & $\begin{array}{c}3 \times 10 \text { followed } \\
\text { by } 1 \times 15\end{array}$ & $\begin{array}{c}10 \\
\min \\
\end{array}$ \\
\hline 10 & As for phase 7 & $4 \times 10$ & $\begin{array}{c}10 \\
\min \end{array}$ \\
\hline 11 & As for phase 7 & $\begin{array}{c}4 \times 10 \text { followed } \\
\text { by } 1 \times 15\end{array}$ & $\begin{array}{c}10 \\
\min \end{array}$ \\
\hline 12 & As for phase 7 & $5 \times 10$ & $\begin{array}{c}10 \\
\min \end{array}$ \\
\hline
\end{tabular}

All single-leg exercises were performed with both the left and the right legs. The step height was $145 \mathrm{~mm}$.

Postoperative exercises were started immediately after surgery as showed in (Table 2.4); the patients were walking with the assistance of a pair of crutches without touching the ground, after two weeks, walking with the heels contacting the ground was allowed; with the fourth week partial weightbearing was allowed as tolerated by the patient. After six weeks, full-weight bearing on a crutch. The patients walked without support after three months (Sarman et al., 2019). The patient received 6 sessions /week.

Table 2.4: Postoperative exercise program that all groups were done. 


\begin{tabular}{|c|c|c|c|c|}
\hline Knee parameters / Exercises & $\begin{array}{c}0-2 \\
\text { weeks }\end{array}$ & $\begin{array}{c}3-6 \\
\text { weeks }\end{array}$ & $\begin{array}{c}7-12 \\
\text { weeks }\end{array}$ & $\begin{array}{c}13- \\
17 / 22 \\
\text { weeks }\end{array}$ \\
\hline \multicolumn{5}{|l|}{ ROM and flexibility } \\
\hline $\begin{array}{l}\text { Heel slides (+/-slider board, up } \\
\text { wall) }\end{array}$ & 0 & ○ & & \\
\hline $\begin{array}{l}\text { Seated active assisted knee } \\
\text { flexion }\end{array}$ & ○ & ○ & & \\
\hline $\begin{array}{l}\text { Seated calf and hamstring } \\
\text { stretches }\end{array}$ & ○ & ○ & & \\
\hline $\begin{array}{l}\text { Passive extension with roll under } \\
\text { heel }\end{array}$ & & 0 & & \\
\hline Prone hangs (leg off bed) & & 0 & & \\
\hline $\begin{array}{l}\text { Prone assisted knee flexion (belt, } \\
\text { opposite leg) }\end{array}$ & & ○ & & \\
\hline $\begin{array}{l}\text { Stationary bike (high seat } 1 / 2 \\
\text { circles forward/backward } \rightarrow \text { full } \\
\text { circles } \rightarrow \text { lower seat as tolerate }\end{array}$ & & ○ & ○ & \\
\hline $\begin{array}{l}\text { Joint mobilizations (patellar, tib- } \\
\text { femoral) }\end{array}$ & & & ○ & \\
\hline Quad stretches & & & (0) & \\
\hline $\begin{array}{l}\text { Standing weight-bearing calf } \\
\text { stretches: gastroc, soleus }\end{array}$ & & & ○ & \\
\hline \multicolumn{5}{|l|}{$\begin{array}{l}\text { Muscle strength and endurance } \\
\text { quadriceps }\end{array}$} \\
\hline Isometric quads & O & ○ & & \\
\hline Quad over roll & & ○ & & \\
\hline $\begin{array}{l}\text { Closed chain terminal extension } \\
\text { with tubing: forward and } \\
\text { backward facing }\end{array}$ & & ○ & ○ & \\
\hline $\begin{array}{l}\text { Squats: wall, mini, } 60^{\circ}-90^{\circ}(+/- \\
\text { wall) }\end{array}$ & & & (-) & ○ \\
\hline $\begin{array}{l}\text { Shuttle: leg press \& calf press - } 2 \\
\text { legs, } 1 \text { leg (progress with } \uparrow \\
\text { resistance/reps) }\end{array}$ & & & 0 & 0 \\
\hline $\begin{array}{l}\text { Sit to stand: high seat, low seat, } \\
2 \text { legs, single leg }\end{array}$ & & & ○ & ○ \\
\hline Leg press machine: $2-1$ leg & & & 0 & 0 \\
\hline
\end{tabular}




\begin{tabular}{|l|c|c|c|c|}
\hline $\begin{array}{l}\text { Bungee cord walking: forward, } \\
\text { backward, side step, lunging }\end{array}$ & & & $\odot$ & $\odot$ \\
\hline Static Lunge: $1 / 4-1 / 2$-full, dynamic & & & $\odot$ & $\odot$ \\
\hline $\begin{array}{l}\text { Step ups (concentric):5-10-15-20 } \\
\text { cm }\end{array}$ & & & $\odot$ & $\odot$ \\
\hline $\begin{array}{l}\text { Step down (eccentric): } 5-10-15- \\
20 \text { cm }\end{array}$ & & & $\odot$ & $\odot$ \\
\hline Hamstrings / Gluteals: & & & & \\
\hline $\begin{array}{l}\text { Gluteal squeezes (supine or } \\
\text { standing) }\end{array}$ & $\odot$ & $\odot$ & & \\
\hline $\begin{array}{l}\text { Standing hip flexion/extension, } \\
\text { abduction/adduction }\end{array}$ & $\odot$ & $\odot$ & $\odot$ & \\
\hline $\begin{array}{l}\text { Supine straight leg raise x four } \\
\text { directions }\end{array}$ & & $\odot$ & & \\
\hline S/L: clam shells & & $\odot$ & & \\
\hline Prone knee flexion & & $\odot$ & & \\
\hline Quadruped fire hydrant & $\odot$ & $\odot$ & $\odot$ \\
\hline $\begin{array}{l}\text { Supine bridging: double, single, } \\
\text { ball, +knee flexion }\end{array}$ & & & \\
\hline
\end{tabular}

\begin{tabular}{|c|c|c|c|c|}
\hline Knee parameters / Exercises & $\begin{array}{c}0-2 \\
\text { weeks }\end{array}$ & $\begin{array}{c}3-6 \\
\text { weeks }\end{array}$ & $\begin{array}{c}7-12 \\
\text { weeks }\end{array}$ & $\begin{array}{c}13- \\
17 / 22 \\
\text { weeks }\end{array}$ \\
\hline $\begin{array}{l}\text { Hamstring curls: prone, sitting, } \\
\text { standing }\end{array}$ & & & (0) & 0 \\
\hline Chair walking/stool pulls & & & 0 & O \\
\hline $\begin{array}{l}\text { Hip strengthening: weights, } \\
\text { pulleys, tubing }\end{array}$ & & & ○ & (0) \\
\hline Tubing kickbacks (mule kicks) & & & (0) & 0 \\
\hline $\begin{array}{l}\text { Shuttle standing kickbacks } \\
\text { (hip/knee extension) }\end{array}$ & & & & O \\
\hline \multicolumn{5}{|l|}{ Calves } \\
\hline Ankle pumping $+/$ - leg elevation & O & & & \\
\hline Plantar flexion with theraband & & 0 & & \\
\hline Calf raises: $2-1$ foot & & & 0 & 0 \\
\hline Up on toes walking & & & 0 & 0 \\
\hline $\begin{array}{l}\text { Eccentrics calves - heels drops } \\
2-1 \text { leg }\end{array}$ & & & & ○ \\
\hline Proprioception & & & & \\
\hline
\end{tabular}




\begin{tabular}{|c|c|c|c|}
\hline Weight shifting (weigh scales) & & O & \\
\hline $\begin{array}{l}\text { Wobble boards, } 1 / 2 \text { foam roller, } \\
\text { double, single leg }\end{array}$ & & O & 0 \\
\hline $\begin{array}{l}\text { Squats, lunges on dynadisc, } \\
\text { airex, bosu... }\end{array}$ & & () & 0 \\
\hline $\begin{array}{l}\text { Single leg balance, time, } \\
\text { complexity of skill }\end{array}$ & & O & 0 \\
\hline $\begin{array}{l}\text { Standing 747s: eyes open, eyes } \\
\text { closed, on mini tramp }\end{array}$ & & ○ & \\
\hline $\begin{array}{l}\text { Balance training with upper body } \\
\text { patterning for sport }\end{array}$ & & ○ & \\
\hline \multicolumn{4}{|l|}{ Cardiovascular fitness } \\
\hline Bike & 0 & 0 & O \\
\hline Elliptical trainer & & 0 & 0 \\
\hline $\begin{array}{l}\text { Treadmill: forward, backward, } \\
\text { jog, run }\end{array}$ & & & O \\
\hline Sport specific training drills & & & O \\
\hline
\end{tabular}

\subsubsection{Tools:}

In order to achieve the aim of the study, the following measuring parameters were performed: Height $(\mathrm{m})$ using restameter, body weight $(\mathrm{kg})$ using medical scale, the body mass index $(\mathrm{BMI})\left(\mathrm{kg} / \mathrm{m}^{2}\right)$ (Misra and Dhurandhar 2019), (right and left) leg length $(\mathrm{cm})$ using measuring tape, knee movement range $\left({ }^{\circ}\right.$ ) using the goniometer (Hancock et al., 2018), $Y$ balance test (YBT), that is applicable to provide an accurate assessment of the lower limb neuromuscular control. The individual should apply six times on each lower limb in each of the three directions before the main test. Must wear athletic shoes during the test and support foot positioned in the center of the $Y$ balance test platform, leaning against the starting point. The maximum reach distance is measured by the measuring tape, at the point where the foot has reached (Neves et al., 2017).

\section{Results and discussion}

No study participant left the research project for any reason. No side effects or complications were observed during the treatment.

Data collected using different measuring tools, revealed that there were equally maximum improvements in knee measurements in injured leg compared to healthy leg for the three experimental groups with different three exercise rehabilitation programs. The third experimental groups were reached to maximum improvements in $4+17$ weeks followed by first group 
4+20 weeks ended with second group 4+22 weeks. Results were presented in Tables 3.4-3.9 and Figures 3.1and 3.2.

To the best of our knowledge, this investigation is the first to examine the use of mechanical unloading or "un-weighting" technology and aquatherapy to support the reducing time of injured limb efficient restoration before and after low knee osteotomy surgery. Our results suggest that this combined method can be safely and successfully used to promote the speed of restoration before and after surgery without exacerbation of joint symptoms, and resulted in a significant decrease in knee joint pain, improved knee joint function, as well as a substantial increase in thigh muscle strength about the affected knee.

The patients who plan to undergo knee osteotomy surgery, preoperative rehabilitation program should be performed to enhance postsurgical outcomes. Rehabilitation should start as soon as possible after diagnosis (Filbay and Grindem 2019). Preoperative rehabilitation should be included knee extension range-of-motion and quadriceps strength should be specifically targeted as these factors are associated with poor post-surgical outcomes (Melick et al., 2016).

The study results is in line with Peeler et al. (2015) who reported that, mechanical unloading or "un-weighting" is a safe mode of exercise that can be successfully used to decrease knee joint pain, improve joint function, and rise knee muscle strength. This result recommends that the reciprocal nature of muscle contraction observed during the low-load exercises helped a healthy balance in agonist/ antagonist strength about the knee and may improve functional capacity during the daily living activities.

A mechanical unloading or "un-weighting" can be a useful method in the rapidly transition from early postoperative phases up to the high phase of rehabilitation. The benefits of unloading are allowing early initiation of weight bearing and closed kinetic chain activities in a safe environment for healing tissue. Increased vertical ground reaction force and vertical loading have been linked with increased risk for stress injury or overuse injury. Declining these loads will allow safe acceleration of functional activity (Draovitch et al., 2012).

Aquatic therapy results are in line with Kutzner et al. (2017); who reported that the aquatic therapy are frequently recommended for rehabilitation in order to enable mobilization and improve the muscle strengthening while minimizing the joint loads of the lower limb.

According to Michaels and Stevens (2019) study results, the cardiovascular function can also be enhanced as a result of aquatic therapy. Also declining the impact on sore joints will always be beneficial, allowing 
individuals with knee deficient to exercise longer, building endurance, and eventually enhancing strength. This exercise in the water, when done with time, should eventually help the patients to move, not only better through the water, but also more efficiently through the air.

Rahmann (2010) study results, reported that when people with knee deficient find exercising in water an enjoyable and less painful activity, the compliance may be enhanced. The pool environment, with numbers of people exercising together, can be a positive environment and may have an effect on compliance and adherence to exercise for people with knee deficient, over and above the exercise program itself.

Wound healing is a complex process that needs understanding of different factors influencing it to improve healing times of wounds (Khalil et al., 2015).

patients must wait until two weeks after surgery to allow for wounds to heal and this may be slightly varied according to quality of individuals healing (Villalta and Peiris 2013). Depending on this rationale, the knee aquatic therapy rehabilitation time may be delaying than the other study rehabilitation programs.

Table 3.4. Mean, \pm SD and difference\% between different experimental groups for demographic characteristics before and after the knee surgery and treating programs.

\begin{tabular}{|c|c|c|c|c|c|c|c|c|c|c|}
\hline \multirow[b]{2}{*}{$\begin{array}{l}\text { Varia } \\
\text { bles }\end{array}$} & \multicolumn{3}{|c|}{ Group 1} & \multicolumn{3}{|c|}{ Group 2} & \multicolumn{3}{|c|}{ Group 3} & \multirow[b]{2}{*}{$\begin{array}{l}\text { Sk } \\
\text { ew }\end{array}$} \\
\hline & $\begin{array}{l}\text { Bef } \\
\text { ore }\end{array}$ & $\begin{array}{l}\text { Aft } \\
\text { er }\end{array}$ & $\begin{array}{c}\text { Differ } \\
\text { ence } \\
\%\end{array}$ & $\begin{array}{l}\text { Bef } \\
\text { ore }\end{array}$ & $\begin{array}{l}\text { Aft } \\
\text { er }\end{array}$ & $\begin{array}{c}\text { Differ } \\
\text { ence } \\
\%\end{array}$ & $\begin{array}{l}\text { Bef } \\
\text { ore }\end{array}$ & $\begin{array}{l}\text { Aft } \\
\text { er }\end{array}$ & $\begin{array}{c}\text { Differ } \\
\text { ence } \\
\%\end{array}$ & \\
\hline Age & $\begin{array}{c}52.3 \\
8 \\
\pm \\
4.31\end{array}$ & $\begin{array}{c}52 . \\
38 \\
\pm \\
4.3 \\
1\end{array}$ & 0.00 & $\begin{array}{c}53.0 \\
0 \\
\pm \\
4.54\end{array}$ & $\begin{array}{c}53 . \\
00 \\
\pm \\
4.5 \\
4\end{array}$ & 0.00 & $\begin{array}{c}51.7 \\
5 \\
\pm \\
5.65\end{array}$ & $\begin{array}{c}51 . \\
75 \\
\pm \\
5.6 \\
5\end{array}$ & 0.00 & $\begin{array}{c}0.4 \\
5\end{array}$ \\
\hline $\begin{array}{l}\text { Heigh } \\
\text { t (m) }\end{array}$ & $\begin{array}{r}1.74 \\
\pm \\
0.06\end{array}$ & $\begin{array}{c}1.7 \\
6 \\
\pm \\
0.0 \\
6\end{array}$ & 1.15 & $\begin{array}{c}1.76 \\
\pm \\
0.05\end{array}$ & $\begin{array}{c}1.7 \\
8 \\
\pm \\
0.0 \\
5\end{array}$ & 1.14 & $\begin{array}{r}1.75 \\
\pm \\
0.06\end{array}$ & $\begin{array}{c}1.7 \\
7 \\
\pm \\
0.0 \\
6\end{array}$ & 1.14 & $\begin{array}{c}0.1 \\
5\end{array}$ \\
\hline & $\begin{array}{c}85.0 \\
0\end{array}$ & $\begin{array}{l}81 . \\
00\end{array}$ & -4.71 & $\begin{array}{c}83.3 \\
8\end{array}$ & $\begin{array}{l}80 . \\
06\end{array}$ & -3.98 & $\begin{array}{c}86.6 \\
3\end{array}$ & $\begin{array}{l}83 . \\
00\end{array}$ & -4.19 & \\
\hline
\end{tabular}




\begin{tabular}{|c|c|c|c|c|c|c|c|c|c|c|}
\hline $\begin{array}{c}\text { Weig } \\
\text { ht } \\
(\mathrm{kg})\end{array}$ & 7.95 & $\begin{array}{c} \pm \\
7.2 \\
7\end{array}$ & & $\stackrel{ \pm}{9.49}$ & $\begin{array}{c} \pm \\
8.9 \\
5\end{array}$ & & 6.16 & $\begin{array}{c} \pm \\
7.1 \\
5\end{array}$ & & $\begin{array}{c}- \\
0.5 \\
6\end{array}$ \\
\hline $\begin{array}{l}\text { Body- } \\
\text { mass } \\
\text { index } \\
(\mathrm{kg} / \mathrm{m}\end{array}$ & $\begin{array}{c}27.9 \\
7 \\
\pm \\
1.82\end{array}$ & $\begin{array}{c}26 . \\
03 \\
\pm \\
1.8 \\
8\end{array}$ & -6.94 & $\begin{array}{c}26.9 \\
7 \\
\pm \\
2.75\end{array}$ & $\begin{array}{c}25 . \\
32 \\
\pm \\
2.4 \\
6\end{array}$ & -6.12 & $\begin{array}{c}28.2 \\
2 \\
\pm \\
1.71\end{array}$ & $\begin{array}{c}26 . \\
50 \\
\pm \\
2.1 \\
9\end{array}$ & -6.09 & $\begin{array}{c}0.0 \\
1\end{array}$ \\
\hline $\begin{array}{l}\text { Healt } \\
\text { hy } \\
\text { leg } \\
\text { lengt } \\
\text { h } \\
(\mathrm{cm})\end{array}$ & $\begin{array}{c}93.0 \\
0 \\
\\
\pm \\
3.85\end{array}$ & $\begin{array}{c}93 . \\
00 \\
\pm \\
3.8 \\
5\end{array}$ & 0.00 & $\begin{array}{c}93.7 \\
5 \\
\pm \\
3.22\end{array}$ & $\begin{array}{c}93 . \\
75 \\
\pm \\
3.2 \\
2\end{array}$ & 0.00 & $\begin{array}{c}93.5 \\
0 \\
\pm \\
4.35\end{array}$ & $\begin{array}{c}93 . \\
50 \\
\pm \\
4.3 \\
5\end{array}$ & 0.00 & $\begin{array}{c}0.1 \\
3\end{array}$ \\
\hline $\begin{array}{l}\text { Injure } \\
\text { d leg } \\
\text { lengt } \\
\text { h } \\
(\mathrm{cm})\end{array}$ & $\begin{array}{c}88.6 \\
9 \\
\pm \\
3.69\end{array}$ & $\begin{array}{c}92 . \\
06 \\
\pm \\
3.9 \\
4\end{array}$ & 3.80 & $\begin{array}{c}89.3 \\
1 \\
\pm \\
3.10\end{array}$ & $\begin{array}{c}92 . \\
81 \\
\pm \\
3.3 \\
1\end{array}$ & 3.92 & $\begin{array}{c}89.3 \\
1 \\
\pm \\
4.68\end{array}$ & $\begin{array}{c}92 . \\
38 \\
\pm \\
4.5 \\
0\end{array}$ & 3.44 & $\begin{array}{c}0.1 \\
1\end{array}$ \\
\hline
\end{tabular}

Group 1: Mechanical unweighting program

Group 2: Aquatherapy program

Group 3: Combined program

Skew: between different experimental groups before the knee surgery and treating programs for symmetry

Table 3.5. Mean, $\pm S D$, difference between measuring time and difference (\%) between injured and healthy knee flexion of different experimental groups with different measuring time and difference and difference (\%) between legs.

\begin{tabular}{|c|c|c|c|c|c|c|c|c|c|c|c|}
\hline \multirow{2}{*}{$\begin{array}{l}\text { Sug } \\
\text { gest } \\
\text { ed } \\
\text { prog } \\
\text { ram }\end{array}$} & \multirow[b]{2}{*}{$\begin{array}{l}\text { Measuring } \\
\text { time }\end{array}$} & \multicolumn{4}{|c|}{ Injured knee flexion } & \multicolumn{4}{|c|}{ Healthy knee flexion } & \multirow{2}{*}{$\begin{array}{c}\text { Differen } \\
\text { ce } \\
\text { betwee } \\
\mathbf{n} \\
\text { legs }\end{array}$} & \multirow{2}{*}{$\begin{array}{c}\text { Diffe } \\
\text { renc } \\
e \\
(\%)\end{array}$} \\
\hline & & Mean & \pm SD & $\begin{array}{c}\text { Difference } \\
\text { between } \\
\text { measuring } \\
\text { time } \\
\end{array}$ & $\begin{array}{c}\text { Differen } \\
\text { ce } \\
(\%)\end{array}$ & Mean & $\pm \mathrm{SD}$ & $\begin{array}{c}\text { Difference } \\
\text { between } \\
\text { measuring } \\
\text { time } \\
\end{array}$ & $\begin{array}{c}\text { Differ } \\
\text { ence } \\
(\%)\end{array}$ & & \\
\hline \multirow{3}{*}{$\begin{array}{c}\text { Grou } \\
\text { p } 1\end{array}$} & Measure 1 & 102.63 & 6.14 & -28.94 & -28.20 & 46.88 & 3.36 & -10.82 & $\begin{array}{c}- \\
23.08 \\
\end{array}$ & 55.75 & $\begin{array}{c}- \\
54.32 \\
\end{array}$ \\
\hline & Measure2 & 73.69 & 3.40 & -22.44 & -30.45 & 36.06 & 2.62 & -4.18 & 11.59 & 37.63 & $\begin{array}{c}- \\
51.07\end{array}$ \\
\hline & Measure 3 & 51.25 & 2.88 & -19.69 & -38.42 & 31.88 & 2.74 & -3.07 & -9.63 & 19.37 & 3780 \\
\hline
\end{tabular}




\begin{tabular}{|c|c|c|c|c|c|c|c|c|c|c|c|}
\hline & Measure 4 & 31.56 & 1.84 & & & 28.81 & 0.75 & & & 2.75 & -8.71 \\
\hline \multirow{4}{*}{$\begin{array}{c}\text { Grou } \\
\text { p } 2\end{array}$} & Measure 1 & 103.25 & 5.09 & -27.37 & -26.51 & 45.88 & 4.26 & -7.07 & $\begin{array}{c}- \\
15.41\end{array}$ & 57.37 & $\begin{array}{c}- \\
55.56\end{array}$ \\
\hline & Measure2 & 75.88 & 6.73 & -24.44 & -32.21 & 38.81 & 3.05 & -7.75 & $\begin{array}{c}- \\
19.97 \\
\end{array}$ & 37.07 & $\begin{array}{c}- \\
48.85 \\
\end{array}$ \\
\hline & Measure 3 & 51.44 & 5.21 & -21.56 & -41.91 & 31.06 & 2.19 & -2.25 & -7.24 & 20.38 & 39.62 \\
\hline & Measure 4 & 29.88 & 1.90 & & & 28.81 & 1.31 & & & 1.07 & -3.58 \\
\hline \multirow{4}{*}{$\begin{array}{c}\text { Grou } \\
\text { p } 3\end{array}$} & Measure 1 & 102.50 & 6.05 & -28.19 & -27.50 & 46.50 & 4.11 & -8.25 & $\begin{array}{c}- \\
17.74\end{array}$ & 56 & $\begin{array}{c}- \\
54.63 \\
\end{array}$ \\
\hline & Measure2 & 74.31 & 4.82 & -23.25 & -31.29 & 38.25 & 1.73 & -7.39 & $\begin{array}{c}- \\
19.32\end{array}$ & 36.06 & 48.53 \\
\hline & Measure 3 & 51.06 & 4.85 & -21.31 & -41.74 & 30.86 & 1.64 & -1.92 & -6.22 & 20.2 & $\begin{array}{c}- \\
39.56\end{array}$ \\
\hline & Measure 4 & 29.75 & 1.20 & & & 28.94 & 1.15 & & & 0.81 & -2.72 \\
\hline
\end{tabular}

Measure 1: Before surgery before the preoperative program (after diagnosis immediately)

Measure 2: Before surgery after the preoperative program (after 4 weeks)

Measure 3: After surgery before the postoperative program

Measure 4: After surgery after the postoperative program (after 2 weeks)

Table 3.6. Mean, $\pm S D$, difference between measuring time and difference (\%) between injured and healthy knee extension of different experimental groups with different measuring time and difference and difference (\%) between legs.

\begin{tabular}{|c|c|c|c|c|c|c|c|c|c|c|c|}
\hline \multirow{2}{*}{$\begin{array}{l}\text { Suggested } \\
\text { program }\end{array}$} & \multirow{2}{*}{$\begin{array}{l}\text { Measuring } \\
\text { time }\end{array}$} & \multicolumn{4}{|c|}{ Injured knee extension } & \multicolumn{4}{|c|}{ Injured knee extension } & \multirow{2}{*}{$\begin{array}{c}\text { Differ } \\
\text { ence } \\
\text { betwe } \\
\text { en } \\
\text { legs } \\
\end{array}$} & \multirow[t]{2}{*}{$\begin{array}{c}\text { Difference } \\
(\%)\end{array}$} \\
\hline & & Mean & \pm SD & $\begin{array}{l}\text { Difference } \\
\text { between } \\
\text { measures }\end{array}$ & $\begin{array}{l}\text { Difference } \\
\quad(\%)\end{array}$ & Mean & $\stackrel{ \pm}{S D}$ & $\begin{array}{c}\text { Differen } \\
\text { ce } \\
\text { between } \\
\text { measure } \\
\text { s }\end{array}$ & $\begin{array}{l}\text { Difference } \\
(\%)\end{array}$ & & \\
\hline \multirow{4}{*}{ Group 1} & Measure 1 & 157.63 & 4.24 & 10.81 & 6.86 & 179.00 & 0.80 & 0.38 & 0.21 & -21.37 & -13.56 \\
\hline & Measure2 & 168.44 & 2.35 & 8.12 & 4.82 & 179.38 & 0.64 & 0.37 & 0.21 & -10.94 & -6.49 \\
\hline & Measure 3 & 176.56 & 2.61 & 3.13 & 1.77 & 179.75 & 0.38 & 0.13 & 0.07 & -3.19 & -1.81 \\
\hline & Measure 4 & 179.69 & 0.46 & & & 179.88 & 0.23 & & & -0.19 & -0.11 \\
\hline \multirow{4}{*}{ Group 2} & Measure 1 & 158.63 & 5.34 & 11.75 & 7.41 & 179.44 & 0.82 & 0.25 & 0.14 & -20.81 & -13.12 \\
\hline & Measure2 & 170.38 & 4.78 & 4.93 & 2.89 & 179.69 & 0.46 & 0.12 & 0.07 & -9.31 & -5.46 \\
\hline & Measure 3 & 175.31 & 3.41 & 3.32 & 1.89 & 179.81 & 0.37 & 0.13 & 0.07 & -4.5 & -2.57 \\
\hline & Measure 4 & 178.63 & 1.33 & & & 179.94 & 0.18 & & & -1.31 & -0.73 \\
\hline \multirow{3}{*}{ Group 3} & Measure 1 & 158.25 & 5.28 & 12.19 & 7.70 & 178.19 & 1.10 & 1.00 & 0.56 & -19.94 & -12.60 \\
\hline & Measure2 & 170.44 & 2.67 & 6.81 & 4.00 & 179.19 & 0.84 & 0.25 & 0.14 & -8.75 & -5.13 \\
\hline & Measure 3 & 177.25 & 2.96 & 1.81 & 1.02 & 179.44 & 0.73 & 0.25 & 0.14 & -2.19 & -1.24 \\
\hline
\end{tabular}


\begin{tabular}{|c|c|c|} 
Measure 4 & 179.06 & 0.73 \\
\hline
\end{tabular}

179.690 .46

$-0.63$

$-0.35$

Measure 1: Before surgery before the preoperative program (after

diagnosis immediately)

Measure 2: Before surgery after the preoperative program (after 4 weeks)

Measure 3: After surgery before the postoperative program

Measure 4: After surgery after the postoperative program (group 1 after 9 weeks, group 2 after 10 weeks and group 3 after 7 weeks)

Table 3.7. Mean, $\pm S D$, difference and difference (\%) between IKLF and HKLF for first group in y balance test three movements with time, difference (\%) between time and IKLF and HKLF composite and the difference (\%) between composite of IKLF and HKLF.

\begin{tabular}{|c|c|c|c|c|c|c|c|c|c|c|c|}
\hline $\begin{array}{l}\text { Measuring } \\
\text { time }\end{array}$ & 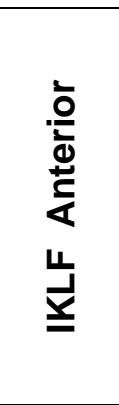 & 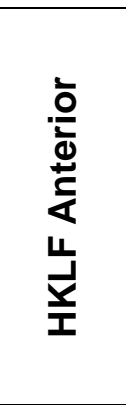 & 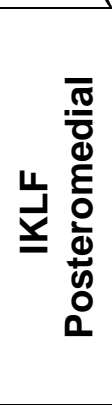 & 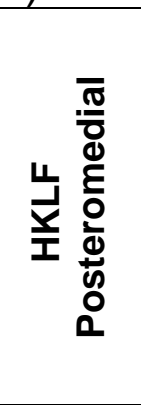 & 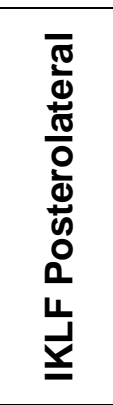 & 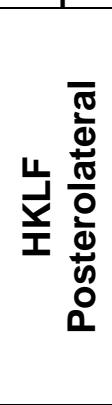 & 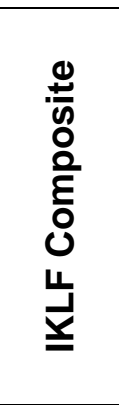 & 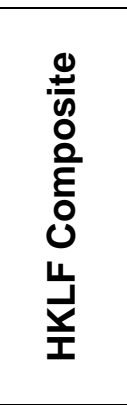 & $\begin{array}{c}\text { difference } \\
(\%) \\
\text { between } \\
\text { different } \\
\text { measuring } \\
\text { time of } \\
\text { IKLF } \\
\text { composite }\end{array}$ & $\begin{array}{c}\text { difference } \\
(\%) \\
\text { between } \\
\text { different } \\
\text { measuring } \\
\text { time of } \\
\text { HKLF } \\
\text { composite }\end{array}$ & $\begin{array}{c}\text { difference } \\
(\%) \\
\text { between } \\
\text { composite } \\
\text { of injurec } \\
\text { and } \\
\text { healthy } \\
\text { knee leg } \\
\text { fulcrum }\end{array}$ \\
\hline \multirow{2}{*}{ Measure 1} & 22.81 & 46.56 & 35.38 & 61.69 & 29.38 & 62.63 & 29.19 & 56.96 & \multirow{2}{*}{16.20} & \multirow{2}{*}{7.76} & \multirow{2}{*}{-95.14} \\
\hline & \pm 2.98 & \pm 4.13 & \pm 2.86 & \pm 4.03 & \pm 2.57 & \pm 4.62 & \pm 2.72 & \pm 4.14 & & & \\
\hline \multirow{2}{*}{ Measure 2} & 26.81 & 49.44 & 41.00 & 67.44 & 33.94 & 67.25 & 33.92 & 61.38 & \multirow{2}{*}{-11.79} & \multirow{2}{*}{3.80} & \multirow{2}{*}{-80.96} \\
\hline & \pm 3.41 & \pm 4.25 & \pm 2.39 & \pm 3.81 & \pm 2.53 & \pm 4.90 & \pm 2.74 & \pm 4.29 & & & \\
\hline \multirow{2}{*}{9 week } & 22.75 & 50.25 & 34.81 & 70.31 & 32.19 & 70.56 & 29.92 & 63.71 & \multirow{2}{*}{45.82} & \multirow{2}{*}{16.87} & \multirow{2}{*}{-112.93} \\
\hline & \pm 2.71 & \pm 5.47 & \pm 1.94 & \pm 5.18 & \pm 2.22 & \pm 6.19 & \pm 2.23 & \pm 5.49 & & & \\
\hline \multirow{2}{*}{10 week } & 32.56 & 55.19 & 49.13 & 84.06 & 49.19 & 84.13 & 43.63 & 74.46 & \multirow{2}{*}{17.03} & \multirow{2}{*}{6.94} & \multirow{2}{*}{-70.66} \\
\hline & \pm 4.44 & \pm 7.05 & \pm 2.84 & \pm 4.44 & \pm 3.47 & \pm 6.85 & \pm 3.46 & \pm 5.94 & & & \\
\hline \multirow{2}{*}{11 week } & 38.06 & 57.94 & 57.88 & 91.44 & 57.25 & 89.50 & 51.06 & 79.63 & \multirow{2}{*}{29.26} & \multirow{2}{*}{4.71} & \multirow{2}{*}{-55.95} \\
\hline & \pm 5.01 & \pm 7.00 & \pm 3.45 & \pm 5.17 & \pm 4.22 & \pm 6.87 & \pm 4.16 & \pm 6.15 & & & \\
\hline \multirow{2}{*}{12 week } & 49.25 & 61.44 & 75.00 & 95.38 & 73.75 & 93.31 & 66.00 & 83.38 & \multirow{2}{*}{9.56} & \multirow{2}{*}{0.47} & \multirow{2}{*}{-26.33} \\
\hline & \pm 6.34 & \pm 7.14 & \pm 4.67 & \pm 4.99 & \pm 5.30 & \pm 6.75 & \pm 5.36 & \pm 6.14 & & & \\
\hline \multirow{2}{*}{13 week } & 53.50 & 61.69 & 82.44 & 95.81 & 81.00 & 93.81 & 72.31 & 83.77 & \multirow{2}{*}{7.66} & \multirow{2}{*}{0.73} & \multirow{2}{*}{-15.85} \\
\hline & \pm 6.42 & \pm 7.19 & \pm 5.04 & \pm 4.90 & \pm 5.81 & \pm 6.70 & \pm 5.63 & \pm 6.12 & & & \\
\hline \multirow{2}{*}{14 week } & 57.63 & 62.19 & 88.75 & 96.38 & 87.19 & 94.56 & 77.85 & 84.38 & \multirow{2}{*}{5.36} & & \\
\hline & \pm 6.85 & \pm 7.28 & \pm 5.40 & \pm 4.71 & \pm 6.28 & \pm 6.82 & \pm 6.07 & \pm 6.10 & & 0.81 & \\
\hline 15 wagk & 60.69 & 62.69 & 93.56 & 97.44 & 91.81 & 95.06 & 82.02 & 85.06 & 1 & & \\
\hline 15 week & \pm 7.14 & \pm 7.44 & \pm 5.55 & \pm 4.90 & \pm 6.76 & \pm 6.79 & \pm 6.35 & \pm 6.20 & .7 & 0.5 & 0.1 \\
\hline 1 & 61.50 & 63.13 & 94.56 & 98.13 & 92.75 & 95.44 & 82.94 & 85.56 & & & \\
\hline 1 & \pm 7.38 & \pm 7.55 & \pm 5.67 & \pm 5.32 & \pm 7.01 & \pm 6.85 & \pm 6.57 & \pm 6.39 & & 2 & \\
\hline 17 week & 62.75 & 63.44 & 96.50 & 98.31 & 94.88 & 96.00 & 84.71 & 85.92 & & & \\
\hline 17 week & \pm 7.61 & \pm 7.64 & \pm 5.76 & \pm 5.30 & \pm 7.17 & \pm 6.92 & \pm 6.74 & \pm 6.43 & 2.36 & 2.6 & -1.40 \\
\hline & 64.38 & 65.63 & 98.94 & 100.81 & 96.81 & 98.06 & 86.71 & 88.17 & & & \\
\hline 1 & \pm 7.68 & \pm 7.02 & \pm 5.85 & \pm 4.83 & \pm 7.06 & \pm 6.55 & \pm 6.74 & \pm 5.95 & & 1.24 & \\
\hline 19 week & 64.88 & 65.81 & 99.44 & 101.00 & 97.25 & 98.31 & 87.19 & 88.38 & -0.22 & 0.00 & -1.36 \\
\hline
\end{tabular}




\begin{tabular}{|c|c|c|c|c|c|c|c|c|c|c|c|}
\hline & \pm 7.77 & \pm 7.07 & \pm 5.60 & \pm 4.82 & \pm 6.80 & \pm 6.44 & \pm 6.62 & \pm 5.95 & & & \\
\hline \multirow{2}{*}{ 20 week } & 64.75 & 65.81 & 99.19 & 101.00 & 97.06 & 98.31 & 87.00 & 88.38 & & & \multirow{2}{*}{1.59} \\
\cline { 2 - 11 } & \pm 7.95 & \pm 7.07 & \pm 5.83 & \pm 4.82 & \pm 7.01 & \pm 6.44 & \pm 6.80 & \pm 5.95 & & & \\
\hline
\end{tabular}

IKLF: Injured knee leg fulcrum, HKLF: Healthy knee leg fulcrum

Table 3.8. Mean, $\pm S D$, difference and difference (\%) between IKLF and HKLF for second group in y balance test three movements with time, difference (\%) between time and IKLF and HKLF composite and the difference (\%) between composite of IKLF and HKLF.

\begin{tabular}{|c|c|c|c|c|c|c|c|c|c|c|c|}
\hline $\begin{array}{l}\text { Measuring } \\
\text { time }\end{array}$ & 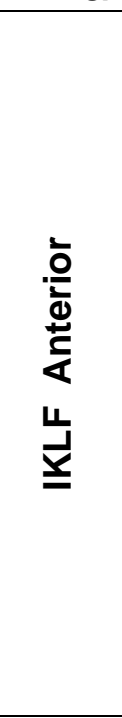 & 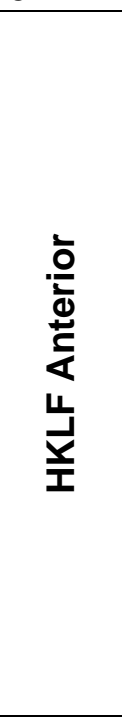 & 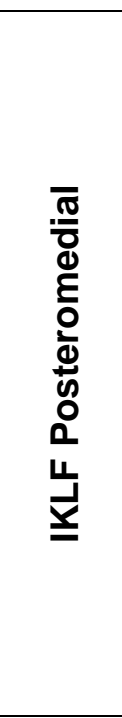 & 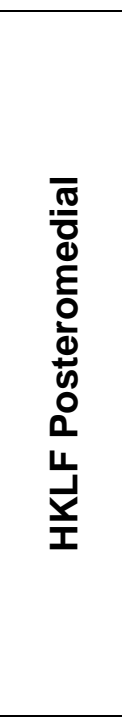 & 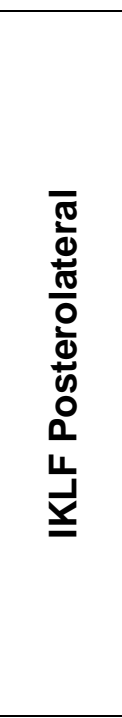 & 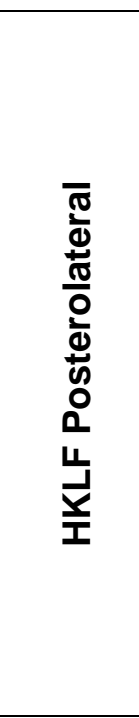 & 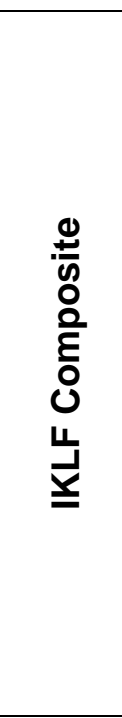 & 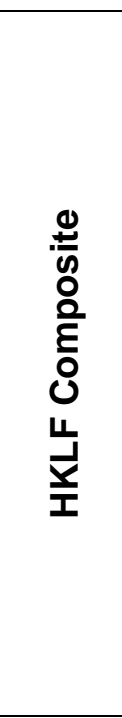 & $\begin{array}{c}\text { difference } \\
(\%) \\
\text { between } \\
\text { different } \\
\text { measuring } \\
\text { time of } \\
\text { IKLF } \\
\text { composite }\end{array}$ & $\begin{array}{c}\text { difference } \\
(\%) \\
\text { between } \\
\text { different } \\
\text { measuring } \\
\text { time of } \\
\text { HKLF } \\
\text { composite }\end{array}$ & $\begin{array}{c}\text { differ } \\
\text { ence } \\
(\%) \\
\text { betw } \\
\text { een } \\
\text { comp } \\
\text { osite } \\
\text { of } \\
\text { injure } \\
\text { d and } \\
\text { healt } \\
\text { hy } \\
\text { knee } \\
\text { leg } \\
\text { fulcr } \\
\text { um }\end{array}$ \\
\hline \multirow{2}{*}{ Measure 1} & 21.63 & 44.31 & 35.25 & 58.88 & 27.63 & 60.44 & 28.17 & 54.54 & \multirow{2}{*}{15.44} & \multirow{2}{*}{14.98} & \multirow{2}{*}{93.61} \\
\hline & \pm 3.67 & \pm 3.95 & \pm 2.41 & \pm 3.61 & \pm 2.97 & \pm 4.35 & \pm 2.33 & \pm 3.54 & & & \\
\hline \multirow{2}{*}{ Measure 2} & 25.56 & 51.00 & 40.00 & 68.50 & 32.00 & 68.63 & 32.52 & 62.71 & \multirow{2}{*}{-8.06} & \multirow{2}{*}{4.54} & \multirow{2}{*}{$\begin{array}{c}- \\
92.84\end{array}$} \\
\hline & \pm 3.44 & \pm 4.02 & \pm 2.31 & \pm 3.67 & \pm 2.42 & \pm 4.54 & \pm 2.02 & \pm 3.70 & & & \\
\hline \multirow[b]{2}{*}{12 week } & 23.13 & 53.00 & 37.25 & 72.31 & 29.31 & 71.38 & 29.90 & 65.56 & \multirow[b]{2}{*}{50.37} & \multirow[b]{2}{*}{1.43} & \multirow{2}{*}{$\begin{array}{c}- \\
119.2 \\
6\end{array}$} \\
\hline & \pm 3.43 & \pm 7.50 & \pm 2.42 & \pm 4.78 & \pm 2.90 & \pm 10.00 & \pm 2.34 & \pm 4.78 & & & \\
\hline \multirow{2}{*}{13 week } & 33.00 & 53.69 & 51.88 & 73.69 & 50.00 & 72.13 & 44.96 & 66.50 & \multirow{2}{*}{19.68} & \multirow{2}{*}{3.82} & \multirow[b]{2}{*}{47.91} \\
\hline & \pm 4.37 & \pm 7.20 & \pm 3.10 & \pm 5.11 & \pm 3.37 & \pm 10.18 & \pm 2.56 & \pm 4.83 & & & \\
\hline \multirow{2}{*}{14 week } & 39.38 & 54.94 & 61.81 & 77.31 & 60.25 & 74.88 & 53.81 & 69.04 & \multirow{2}{*}{7.90} & \multirow{2}{*}{3.53} & \multirow[b]{2}{*}{28.30} \\
\hline & \pm 5.22 & \pm 7.85 & \pm 3.46 & \pm 4.55 & \pm 3.85 & \pm 10.43 & \pm 2.91 & \pm 4.84 & & & \\
\hline \multirow{2}{*}{15 week } & 43.06 & 54.94 & 66.44 & 80.13 & 64.69 & 79.38 & 58.06 & 71.48 & \multirow{2}{*}{10.92} & \multirow{2}{*}{3.18} & \multirow[b]{2}{*}{3.11} \\
\hline & \pm 5.77 & \pm 8.70 & \pm 3.75 & \pm 4.22 & \pm 4.01 & \pm 10.63 & \pm 3.16 & \pm 4.62 & & & \\
\hline \multirow{2}{*}{16 week } & 49.25 & 55.88 & 74.94 & 85.06 & 69.00 & 80.31 & 64.40 & 73.75 & \multirow{2}{*}{13.56} & \multirow{2}{*}{4.46} & \multirow{2}{*}{$\begin{array}{c}- \\
14.52\end{array}$} \\
\hline & \pm 6.62 & \pm 8.40 & \pm 4.31 & \pm 3.92 & \pm 4.08 & \pm 6.09 & \pm 3.42 & \pm 4.87 & & & \\
\hline \multirow{2}{*}{17 week } & 55.44 & 56.75 & 85.31 & 89.63 & 78.63 & 84.75 & 73.13 & 77.04 & \multirow{2}{*}{9.82} & \multirow{2}{*}{4.69} & \multirow{2}{*}{-5.35} \\
\hline & \pm 7.45 & \pm 8.37 & \pm 4.80 & \pm 3.71 & \pm 4.68 & \pm 5.75 & \pm 3.87 & \pm 4.68 & & & \\
\hline 18 week & 60.38 & 59.75 & 92.13 & 93.69 & 88.44 & 88.50 & 80.31 & 80.65 & 366 & 43 & 42 \\
\hline to week & \pm 8.13 & \pm 8.50 & \pm 5.53 & \pm 3.99 & \pm 5.17 & \pm 5.57 & \pm 4.26 & \pm 4.59 & 3.66 & 4.3 & 0.42 \\
\hline 19 week & 62.25 & 63.19 & 95.13 & 97.25 & 92.38 & 91.94 & 83.25 & 84.13 & 233 & 262 & -106 \\
\hline 19 Week & \pm 8.29 & \pm 8.77 & \pm 5.60 & \pm 4.41 & \pm 5.26 & \pm 5.43 & \pm 4.42 & \pm 4.48 & 2.33 & 2.62 & -1.00 \\
\hline & 63.44 & 64.44 & 97.06 & 98.38 & 95.06 & 96.19 & 85.19 & 86.33 & & 0 & -134 \\
\hline K & \pm 8.49 & \pm 8.59 & \pm 5.48 & \pm 5.41 & \pm 5.10 & \pm 4.96 & \pm 4.28 & \pm 4.28 & 0.13 & $0 .<4$ & -1.34 \\
\hline
\end{tabular}




\begin{tabular}{|c|c|c|c|c|c|c|c|c|c|c|c|}
\hline \multirow{2}{*}{ 21 week } & 64.13 & 64.75 & 97.75 & 98.56 & 95.56 & 96.31 & 85.81 & 86.54 & \multirow{2}{*}{0.20} & \multirow{2}{*}{0.00} & -0.85 \\
\cline { 2 - 11 } & \pm 8.21 & \pm 8.30 & \pm 5.36 & \pm 5.23 & \pm 5.05 & \pm 5.11 & \pm 4.32 & \pm 4.19 & & \\
\hline \multirow{2}{*}{ 22 week } & 64.25 & 64.75 & 97.94 & 98.56 & 95.75 & 96.31 & 85.98 & 86.54 & & & 0.65 \\
\cline { 2 - 8 } & \pm 8.26 & \pm 8.30 & \pm 5.22 & \pm 5.23 & \pm 4.86 & \pm 5.11 & \pm 4.23 & \pm 4.19 & & & \\
\hline
\end{tabular}

Table 3.9. Mean, $\pm S D$, difference and difference (\%) between IKLF and HKLF for third group in y balance test three movements with time, difference (\%) between time and IKLF and HKLF composite and the difference (\%) between composite of IKLF and HKLF.

\begin{tabular}{|c|c|c|c|c|c|c|c|c|c|c|c|}
\hline $\begin{array}{l}\text { Measuring } \\
\text { time }\end{array}$ & 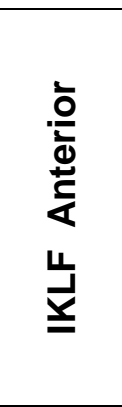 & 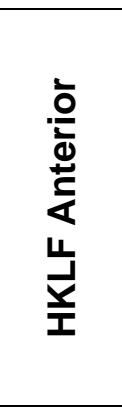 & 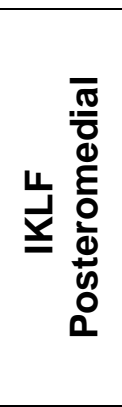 & 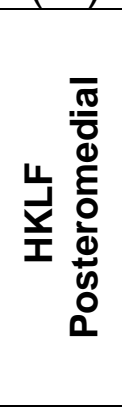 & 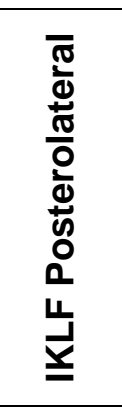 & 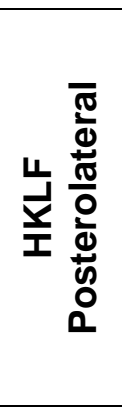 & 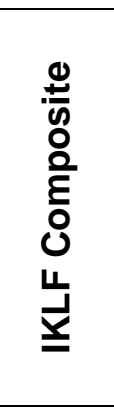 & 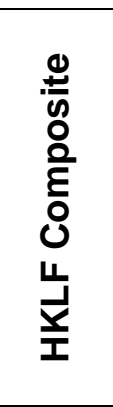 & $\begin{array}{c}\text { difference } \\
(\%) \\
\text { between } \\
\text { different } \\
\text { measuring } \\
\text { time of } \\
\text { IKLF } \\
\text { composite }\end{array}$ & $\begin{array}{l}\text { difference } \\
(\%) \\
\text { between } \\
\text { different } \\
\text { measuring } \\
\text { time of } \\
\text { HKLF } \\
\text { composite }\end{array}$ & $\begin{array}{c}\text { difference } \\
(\%) \\
\text { between } \\
\text { composite } \\
\text { of injured } \\
\text { and } \\
\text { healthy } \\
\text { knee leg } \\
\text { fulcrum }\end{array}$ \\
\hline \multirow{2}{*}{ Measure 1} & 21.00 & 44.44 & 34.69 & 58.13 & 28.00 & 60.19 & 27.90 & 54.25 & \multirow{2}{*}{16.27} & \multirow{2}{*}{14.93} & \multirow[b]{2}{*}{-94.44} \\
\hline & \pm 2.82 & \pm 4.47 & \pm 2.42 & \pm 3.57 & \pm 2.84 & \pm 4.88 & \pm 2.56 & \pm 4.18 & & & \\
\hline \multirow{2}{*}{ Measure 2} & 25.13 & 51.25 & 39.13 & 68.38 & 33.06 & 67.44 & 32.44 & 62.35 & \multirow{2}{*}{-13.38} & \multirow{2}{*}{4.46} & \multirow{2}{*}{-92.20} \\
\hline & \pm 3.29 & \pm 4.48 & \pm 2.53 & \pm 3.95 & \pm 2.60 & \pm 4.73 & \pm 2.75 & \pm 4.30 & & & \\
\hline \multirow{2}{*}{9 week } & 22.00 & 53.06 & 35.19 & 73.25 & 27.13 & 69.06 & 28.10 & 65.13 & \multirow{2}{*}{27.83} & \multirow{2}{*}{2.69} & \multirow{2}{*}{-131.78} \\
\hline & \pm 3.26 & \pm 7.40 & \pm 2.55 & \pm 5.07 & \pm 2.33 & \pm 4.07 & \pm 2.34 & \pm 5.19 & & & \\
\hline \multirow{2}{*}{10 week } & 26.44 & 53.44 & 40.94 & 75.63 & 40.38 & 71.56 & 35.92 & 66.88 & \multirow{2}{*}{31.60} & \multirow{2}{*}{2.99} & \multirow{2}{*}{-86.19} \\
\hline & \pm 3.31 & \pm 7.30 & \pm 1.97 & \pm 5.61 & \pm 3.17 & \pm 4.52 & \pm 2.71 & \pm 5.39 & & & \\
\hline \multirow{2}{*}{11 week } & 35.25 & 54.25 & 53.81 & 77.81 & 52.75 & 74.56 & 47.27 & 68.88 & \multirow{2}{*}{22.91} & \multirow{2}{*}{6.40} & \multirow{2}{*}{-45.72} \\
\hline & \pm 4.52 & \pm 7.49 & \pm 3.16 & \pm 5.81 & \pm 4.03 & \pm 4.89 & \pm 3.79 & \pm 5.70 & & & \\
\hline \multirow{2}{*}{12 week } & 43.38 & 55.50 & 65.13 & 84.81 & 65.81 & 79.56 & 58.10 & 73.29 & \multirow{2}{*}{7.54} & \multirow{2}{*}{4.56} & \multirow{2}{*}{-26.14} \\
\hline & \pm 5.71 & \pm 7.70 & \pm 3.60 & \pm 6.35 & \pm 4.98 & \pm 5.20 & \pm 4.67 & \pm 6.10 & & & \\
\hline \multirow{2}{*}{13 week } & 46.69 & 57.50 & 71.25 & 88.81 & 69.50 & 83.56 & 62.48 & 76.63 & \multirow{2}{*}{14.00} & \multirow{2}{*}{7.11} & \multirow{2}{*}{-22.65} \\
\hline & \pm 6.15 & \pm 8.50 & \pm 4.20 & \pm 6.64 & \pm 5.39 & \pm 5.80 & \pm 5.11 & \pm 6.76 & & & \\
\hline \multirow{2}{*}{14 week } & 53.13 & 61.25 & 81.25 & 93.31 & 79.31 & 91.69 & 71.23 & 82.08 & \multirow{2}{*}{13.63} & \multirow{2}{*}{3.16} & \multirow{2}{*}{-15.23} \\
\hline & \pm 7.06 & \pm 8.54 & \pm 4.68 & \pm 6.06 & \pm 6.05 & \pm 7.52 & \pm 5.76 & \pm 7.14 & & & \\
\hline 15 weok & 60.38 & 63.00 & 92.13 & 96.13 & 90.31 & 94.88 & 80.94 & 84.67 & 553 & 21 & 161 \\
\hline 15 week & \pm 7.92 & \pm 8.27 & \pm 5.50 & \pm 5.60 & \pm 6.83 & \pm 7.41 & \pm 6.54 & \pm 6.85 & & 2.11 & \\
\hline & 63.63 & 64.94 & 97.38 & 98.06 & 95.25 & 96.38 & 85.42 & 86.46 & & & \\
\hline 16 week & \pm 8.37 & \pm 8.57 & \pm 5.46 & \pm 5.47 & \pm 7.29 & \pm 7.66 & \pm 6.82 & \pm 7.02 & 00.16 & 0.00 & -1.22 \\
\hline & 63.88 & 64.94 & 97.44 & 98.06 & 95.38 & 96.38 & 85.56 & 86.46 & & & \\
\hline Neek & \pm 8.45 & \pm 8.57 & \pm 5.40 & \pm 5.47 & \pm 7.38 & \pm 7.66 & \pm 6.85 & \pm 7.02 & & & 1.05 \\
\hline
\end{tabular}


The result of current study shows that use of mechanical unloading or "unweighting" technology combined with aquatherapy can reducing the time of injured limb efficient restoration before and after low knee osteotomy surgery. This was agreed with Lund et al. (2008) study results, reported that the combination of aquatic and land-based exercise should be the preferred exercise regimen in knee rehabilitation. With addition that the land-based exercise must be done using mechanical unloading technology.

Aalderink et al. (2010) study documented that, the complete recovery after knee osteotomy surgery, defined as pain-free return to full activity, including unlimited exercise can take up to 24 weeks or longer after surgery. Our study results were demonstrated that, the third experimental group was reached to the improvement after 4 weeks preoperative rehabilitation program and 17 postoperative rehabilitation program.

\section{Conclusion}

There were equally maximum improvements in knee measurements in injured leg compared to healthy leg for the three experimental groups with different three exercise rehabilitation programs. The third experimental group was reached to maximum improvements after $4+17$ weeks followed by first group 4+20 weeks ended with second group 4+22 weeks. Three experimental groups full flexion were performed at the end of the second week after surgery. Full extension for third, first and second experimental groups was performed at the end of the seven, nine and ten week after surgery respectively.

The combined effect of mechanical unweighting and aqua-therapy on reducing time of injured limb efficient restoration before and after knee osteotomy surgery was the most effective knee rehabilitation program in decreasing the rehabilitation time.

References:

1. Aalderink K.J., Shaffer M. and Amendola A. 2010. Rehabilitation following high tibial osteotomy. Clin Sports Med, 29: 291-301.

2. Adams T., Band-Entrup D., Kuhn S., Legere L., Mace K., Paggi A. and Penney M. 2013. Physical therapy management of knee osteoarthritis in the middle-aged athlete. Sports Med Arthrosc Rev, 21(1).

3. Adnan R., Rafieb M. H., Ahmadb H., Sulaimana N., Ismail S. I. 2014. Effects of land based and water based walking exercise among knee 
osteoarthritis patients. Journal of advanced medical research, 4 (3):112

4. Bennell K. L., Nelligan R. K, Kimp A. J, Wrigley T. V., Metcalf B., Kasza J., Hodges P. W. and Hinman R. S.2019. Comparison of weight bearing functional exercise and non-weight bearing quadriceps strengthening exercise on pain and function for people with knee osteoarthritis and obesity: protocol for the TARGET randomised controlled trial. BMC musculoskeletal disorders.

5. Brinkman J. M., Lobenhoffer P., Agneskirchner J. D., Staubli A. E., Wymenga A. B. and Heerwaarden R. J. 2008. Osteotomies around the knee: Patient selection, stability of flexion and bone healing in high tibial osteotomies. The journal of bone and joint surgery, 9(12).

6. Brouwer R.W., Huizinga M.R., Duivenvoorden T., van Raaij T.M., Verhagen A.P., Bierma-Zeinstra S.M.A. and Verhaar J.A.N.2014. Osteotomy for treating knee osteoarthritis (Review). Cochrane database of systematic reviews, 1.

7. Castrogiovanni P. and Musumeci G.2017. Which is the best physical treatment for osteoarthritis? Journal of functional morphology and kinesiology, 1: 54-68.

8. Draovitch P., Maschi R.t A. and Hettler J. 2012. Return to sport following hip injury. Current review in musculoskeletal medicine, 5(1): 9-14.

9. Fernandes T. L., Pedrinelli A., Hernandez A. J.2011. Muscle injury - physiopathology, diagnosis, treatment and clinical presentation. Sociedade Brasileira de Ortopedia e Traumatologia, 46(3):247-55.

10. Filbay S.R. and Grindem H.. 2019. Evidence-based recommendations for the mangment of antierior cruciate ligament (ACL) rupture. Best practice and clinical rheumatology, 33: 33- 47.

11. Gao L., Madry H., Chugaev D. V., Denti M., Frolov A., Burtsev M., Magnitskaya N., Mukhanov V., Neyret P., Solomin L. N., Sorokin E., Staubli A. E., Stone K. R., Vilenskiy V., Zayats V., Pape D. and Korolev A.2019. Advances in modern osteotomies around the knee. Report on the association of sports traumatology, arthroscopy, orthopaedic surgery, rehabilitation (ASTAOR) Moscow international osteotomy congress 2017. Journal of experimental orthopaedics , 6(9).

12. Hancock G. E., Hepworth T. and Wembridge K. 2018. Accuracy and reliability of knee goniometry methods. Journal of experimental orthopaedics, 5:46.

13. Hinman R. S., Heywood S. E. and Day A. R. 2006. Aquatic physical therapy for hip and knee osteoarthritis: Results of a single- 
blind randomized controlled trial. Journal of American physical therapy association, 87:32-43.

14. Khalil H., Cullen M., Chambers H., Carroll M. and Walker J.2015. Elements affecting wound healing time: An evidence based analysis. Wound Repair and Regeneration, 23: 550-556.

15. Kim D. Y., Hwang J. H. and ParK W. H.2015. Effects of 4 weeks preoperative exercise on knee extensor strength after anterior cruciate ligament reconstruction. Journal of the society of physical therapy science, 27: 2693-2696.

16. Kutzner I., Richter A., Gordt K., Dymke J., Damm P., Duda G. $\mathbf{N}$ and Bergmann G. 2017. Does aquatic exercise reduce hip and knee joint loading? In vivo load measurements with instrumented implants. Plos one.

17. Lieshout W. A. M., Valkering K. P., Koenraadt K. L. M., Jamaludin F. S., Kerkhoffs G. M. M. J. and Geenen R. C. I. 2018. The negative effect of joint line elevation after total knee arthroplasty on outcome. Knee surgery, sports traumatology, arthroscopy.

18. Lund H., Weile U., Christensen R., Rostock B., Downey A., Bartels E. M., Samsøe B. D. and Bliddal H. 2008. A randomized controlled trial of aquatic land- based exercise in patients with knee osteoarthritis. J Rehabil Med, 40: 137-144.

19. Mangione K. K., Axen K. and Hass F. 1996. Mechanical unweighting effects on treadmill exercise and pain in elderly people with osteoarthritis of the knee. Physical Theropy, 76 (4).

20. Melick N., Cingel R. E. H., Brooijmans F., Neeter C., Tienen T., Hullegie W. and Sanden Maria W. G. N. 2016. Evidence-based clinical practice update: practice guidelines for anterior cruciate ligament rehabilitation based on a systematic review and multidisciplinary consensus. Br J Sports Med, 50:1506-151

21. Michaels N.N. and Stevens S. 2019. Aquatic exercise for patients with osteoarthritis. Researches in arthritis and bone study, 1(4).

22. Misra A. and Dhurandhar N. V.2019. Current formula for calculating body mass index is applicable to Asian populations. Nutrition and Diabetes, 9:3.

23. Mooventhan A. and Nivethitha L.2014.Scientific evidence based effects of hydrotherapy on various systems of the body. North American journal of medicine science, 6(5): 199-209. 
24. Nawghare S. and Brooks S.2013. Manipulation under anesthesia for stiffness after total knee replacement: A systematic review. Journal of orthopedics and allied sciences, 1 (1).

25. Neves L. F., Souza C. Q., Stoffel M. and Picasso C. L. M. 2017. The $Y$ Balance Test - How and Why to Do it? International Physical Medicine \& Rehabilitation Journal, 2(4).

26. Peeler J., Christian M., Cooper J., Leiter J. and MacDonald P. 2015. Managing knee osteoarthritis: The effects of body weight supported physical activity on joint pain, function, and thigh muscle strength. Clin J Sport.

27. Rahmann A. E.2019. Exercise for people with hip or knee osteoarthritis: a comparison of land-based and aquatic interventions. Open access journal of sports medicine, 1: 123-135

28. Sarman H., Isik C., Uslu M., Inanmaz M.E.2019. High tibial osteotomy using a locking titanium plate with or without autografting autografting. Acta Ortop Bras, 27(2):80-4.

29. Sarsak H. I. 2.18. Effectiveness of aquatic therapy on reducing pain and increasing physical function in adult patients with hip and/or knee osteoarthritis. Examine in physical medicine and rehabilitation, 2(1):1-4.

30. Sisto S.A. and Malanga G. 2006. Osteoarthritis and therapeutic exercise. American journal of physical medicine and rehabilitation, 85:69-S78.

31. Syed S. and Wani S.2014. Effect of two different manual therapy protocols on osteoartrite knee pain and functional disability: A comparative study .Romanian of Bone and Joint Surgery physical therapy, 20 (34).

32. Villalta M. E. and Peiris L. C. 2013. Early aquatic therapy improves function and does not increase risk of wound-related adverse events for orthopedic surgery: A systematic review and meta-analysis. Archive of physical medicine and rehabilitation, 94:138-48.

33. Ziegler R., Goebel L., Seidel R., Cucchiarini M., Pape D., Madry H. 2015. Effect of open wedge high tibial osteotomy on the lateral tibiofemoral compartment in sheep. Part III: analysis of the microstructure of the subchondral bone and correlations with the articular cartilage and meniscus. Journal of knee surg sports traumatol arthrosc, 23:2704-2714. 